\title{
Médiévales
}

Langues, Textes, Histoire

74 | printemps 2018

Chanter la Croisade albigeoise

\section{Hermogène le rhéteur et Bohémond le bâilleur. Échos de l'École de Hereford dans l'Ipomedon de Hue de Rotelande}

Hermogenes the Rhetorician and Bohemond the Yawner. Echoes from the

Hereford School in Hue de Rotelande's Ipomedon

\section{Vladimir Agrigoroaei}

\section{OpenEdition \\ Journals}

\section{Édition électronique}

URL : https://journals.openedition.org/medievales/8592

DOI : 10.4000/medievales.8592

ISSN : $1777-5892$

\section{Éditeur}

Presses universitaires de Vincennes

\section{Édition imprimée}

Date de publication : 15 juillet 2018

Pagination : 121-132

ISBN : 978-2-84292-837-7

ISSN : 0751-2708

\section{Référence électronique}

Vladimir Agrigoroaei, « Hermogène le rhéteur et Bohémond le bâilleur. Échos de l'École de Hereford dans I'/pomedon de Hue de Rotelande », Médiévales [En ligne], 74 I printemps 2018, mis en ligne le 15 juillet 2019, consulté le 22 avril 2022. URL : http://journals.openedition.org/medievales/8592 ; DOI : https://doi.org/10.4000/medievales.8592 
Vladimir Agrigoroaei

\section{Hermogène le rhéteur et Bohémond le bâilleur Échos de l'École de Hereford dans l'Ipomedon de Hue de Rotelande}

L'Ipomedon de Hue de Rotelande, roman en vers de la fin du XII ${ }^{\mathrm{e}}$ siècle, trouve difficilement sa place parmi les genres littéraires médiévaux tels que nous les concevons aujourd'hui. À la fois parodie du Roman de Thèbes et satire des tout premiers romans arthuriens, l'Ipomedon reprend des thèmes et des motifs d'autres textes littéraires, mais il demeure esthétiquement singulier. Le chevalier protagoniste est le fils d'Hermogenés roi des Pouilles. Amoureux d'une princesse calabraise, il cache son identité et devient son valet. Cependant, il décide de partir à l'étranger pour montrer sa prouesse, tout en revenant pour participer à un tournoi. Il aime la princesse, mais se cache. Il est vainqueur, mais ne le dit jamais. Il peut se marier avec sa future épouse, mais décide sans raison de la quitter. Ipomedon, inconsistant, peut passer pour un antihéros. Le style du roman est souvent parodique, parfois aussi érotique. On utilise quelquefois des mots vulgaires. Hue de Rotelande, son auteur, s'est tellement amusé à créer ces contresens que la critique a souvent considéré - et certains considèrent encore - qu'il s'agit d'une œuvre littéraire assez médiocre ${ }^{1}$.

Néanmoins, l'Ipomedon ne peut pas être jugé selon les règles du roman ou de la chanson épique ; ce n'est qu'un divertissement de clerc. Et les allusions qui tissent la trame narrative renvoient dans des directions parfois surprenantes, inattendues. J'ai déjà remarqué ailleurs ${ }^{2}$ que le nom de

1. L'édition de référence pour ce texte est celle de A. J. HOLDEN : Ipomedon, poème de Hue de Rotelande édité avec introduction, notes et glossaire, Paris, 1979. Pour une ancienne édition, que nous n'utilisons pas, voir Ipomedon, ein französicher Abenteuerroman des 12. Jahrhunderts, éd. E. KölBING et E. Koschwitz, Breslau, 1889. Cf. Ch. H. LivingSTON, « Manuscript fragments of a continental French version of the Roman d'Ipomedon », Modern Philology, 40/2 (1942-1943), p. 117-130.

2. V.AGRIGOROAEI, «Quelle matière pourquelle translatio ?L'héritage sympériphérique de l'Antiquité dans certains textes français du XII ${ }^{\mathrm{e}}$ siècle », dans C. FERLAMPIN-ACHER et 
Tholomeu, précepteur d'Ipomedon, a été repris du Roman de Thèbes, que Tholomeu n'est qu'un masque. Il constitue une allusion évidente à Ptolémée le géographe-astronome, car le Tholomeu livresque de Hue de Rotelande n'est plus un guerrier, mais un savant, précepteur d'un jeune chevalier. Il m'est paru tout autant évident que ce nom d'inspiration antique servait uniquement à proclamer la Sagesse même, transférée par enseignement à son disciple. J'en ai eu une preuve de plus dans le fait que l'auteur n'a pas insisté sur la sagesse de Tholomeu. Le nom de ce dernier et le fait qu'il était polyglotte suffisaient pour donner du poids au personnage ${ }^{3}$. C'est une référence que j’ai définie comme «sympériphérique ».

Dans cette même étude, j'ai également remarqué qu'un autre nom, celui du père du protagoniste, Hermogenés, a été vraisemblablement inspiré du nom d'un rhétoricien grec du tournant du $\mathrm{III}^{\mathrm{e}}$ siècle : Hermogène de Tarse, personnification du jeune sage ${ }^{4}$. Le texte en ancien français dit très peu sur le personnage, qui joue un rôle introductif et ne réapparaît qu'à la fin du roman, lorsqu'on annonce sa mort à Ipomedon ${ }^{5}$ :

En Poile avoit en icel tens

Un reis qi ert de mult grant sens ;

Hermogenés fust apelé,

C. GîrBeA éd., Matières à débat. La notion de matière dans la littérature médiévale, Rennes, 2017, p. 587-602 (p. 590), que nous reprenons dans la partie introductive du présent article. Pour les rapports plus compliqués de l'Ipomedon avec le Roman de Thèbes, voir en dernier lieu F. MORA, «Remploi et sens du jeu dans quelques textes médio-latins et français des $\mathrm{XII}^{\mathrm{e}}$ et XIII ${ }^{\mathrm{e}}$ siècles : Baudri de Bourgueil, Hue de Rotelande, Renaut de Beaujeu », dans M. ZimMERMANN éd., Auctor et auctoritas. Invention et conformisme dans l'écriture médiévale. Actes du colloque tenu à l'Université de Versailles-Saint-Quentin-en-Yvelines (1416 juin 1999), Paris, 2001, p. 219-230. Pour deux études concernant l'anthroponymie dans les romans de Hue de Rotelande, voir M.-L. CHÊNERIE, « Dénomination et anthroponymie dans les romans de Hue de Rotelande », Les Lettres romanes, 52, 3/4 (1998), p. 203-234 ; et M.-L. CHÊNERIE, « La dénomination des personnages féminins dans les romans de Hue de Rotelande », dans J. C. FAUCON, A. LABbÉ et D. QuÉRuel éd., Miscellanea Mediaevalia. Mélanges offerts à Philippe Ménard, Paris, 1998, vol. 1, p. 349-359.

3. HuE DE Rotelande, Ipomedon, v. 325-326 : « Mes Tholomeu esteit mult sages, / Si saveit de plusurs langages », p. 76 .

4. La présence du nom d'Hermogenés dans l'Ipomedon a attiré très peu d'attention. Julien Vinot, dans sa thèse récente, ne lui consacre qu'une note de bas de page où il suppose que ce nom pourrait être inspiré par le dieu antique Hermès ou par Hermogène, disciple de Socrate. J. VINOT, Répétition et variation dans les romans de Hue de Rotelande, thèse de l'Université de Montréal, 2009, p. 247, n. 156: «Peut-être faut-il voir aussi dans ce nom celui du messager des dieux, Hermès, ou celui du philosophe grec, Hermogène, disciple de Socrate. $C f$. « Hermogenés, fondateur de cette race, comme en témoigne la racine latine - gen comprise dans son nom» (même page, texte principal). Julien Vinot suit M.-L. CHÊNERIE, «Dénomination et anthroponymie... », p. 218 : «Hermogenés, nom composé de la racine -gen : race et du nom Hermos, qui doit combiner au moins deux référents, un fondateur de ville, un législateur, et peut-être un rhéteur du V $\mathrm{V}^{\mathrm{e}} \mathrm{s}$. ap. J.-C. »

5. HuE DE Rotelande, Ipomedon, v. 169-174, p. 69, et v. 7209-7212, p. 381. 
Des leis iert cointes e sené,

E fust pruz en chivalerie

E mult iert fundez en clergie [...]

[...] Kar n'aveit pas del tut un meis

Mort fut Hermogenés, li reis,

Sis peres ; pur ço li vunt quere

Pur faire le rei de la terre.

En revanche, ce n'est pas un hasard si le roi est brave et sage. La sagesse ne constitue bien évidemment qu'un lieu commun des portraits de tous les bons rois que l'on rencontre dans les romans médiévaux; mais dans le cas d'Hermogène (et de Tholomeu) on croit reconnaître l'adage nomen est omen ${ }^{6}$. Les sources antiques racontent qu'Hermogène, enfant prodige, a composé plusieurs traités de rhétorique. Atteint par une méningite à l'âge de vingt-cinq ans, il a perdu toute sa sagesse ${ }^{7}$. L'utilisation des noms Tholomeu et Hermogène, porteurs d'une sagesse sans contenu, me semblait donc créer l'image d'un savoir que l'auteur français du XII siècle n'avait pas le temps ou les moyens de définir précisément ${ }^{8}$. Ce savoir devait être le Savoir. Peu importe que l'astronomie de Ptolémée n'ait rien en commun avec la rhétorique d'Hermogène de Tarse. Je n'étais pas parvenu cependant à montrer comment le nom d'Hermogène de Tarse, rhéteur de l'Antiquité grecque, avait survécu un millénaire entier pour faire enfin surface dans un roman anglo-normand de la fin du XII ${ }^{\mathrm{e}}$ siècle.

Cependant, un tel lien existe. Le but de la présente étude est d'avancer l'hypothèse que le nom Hermogenés du roman de Hue de Rotelande constitue une preuve collatérale du fait que l'École de Hereford avait pu être en contact avec les milieux savants de l'Italie du Sud. La Rhétorique d'Hermogène de Tarse était en effet connue dans le milieu savant calabrais du XII ${ }^{\mathrm{e}}$ siècle. Mais, avant de soumettre la preuve ultime de ces rapports au jugement du lecteur, je dois prendre soin de partager avec lui quelques mots introductifs sur Hue de Rotelande et sur l'École de Hereford.

6. Cette idée a été énoncée pour une première fois par M.-L. CHÊNERIE, « Dénomination et anthroponymie... », p. 229, à propos du nom de "Tholomeu".

7. $C f$., à titre d'exemple, M. HEATH, Hermogenes : On issues. Strategies of Argument in Later Greek Rhetoric, Oxford, 1995.

8. Il faut cependant distinguer les deux cas. Il est vrai que le Roman de Thèbes et la Thébaïde sont connus de tous et que Tholomeu est pour ainsi dire un « texte déjà donné ». Hermogène ne bénéficie pas, en revanche, de la même renommée, et son nom n'apparaît pas ailleurs dans la littérature française médiévale. Il ne porte pas avec lui le savoir et le pouvoir d'autres personnages. 


\section{Hermogène le rhéteur}

Hue de Rotelande (Rhuddlan, dans le voisinage du Pays des Galles ?) vivait dans le Herefordshire, plus précisément à neuf kilomètres de la ville de Hereford. Il dit, à la fin de son premier poème, qu'il a une maison à Credenhill. Son deuxième roman (Protheselaus) a été écrit pour un noble local, Guillaume Fitz-Baderon, seigneur de Monmouth. Le premier roman (Ipomedon) n'avait pourtant pas de commanditaire et l'auteur s'est amusé à persifler Gautier Map, peut-être de manière amicale, comme le suppose A. Holden ; en tout cas, il connaissait bien l'auteur du De nugis curialium (Gautier Map a été chanoine de Hereford). Enfin, notre auteur parle aussi d'un certain Hugues de Hongrie, chanoine et maître à Hereford ${ }^{9}$.

À Hereford, les maîtres enseignaient la théologie, la grammaire, la rhétorique, la musique, l'astrologie et l'astronomie. Une importante bibliothèque, $\mathrm{au} \mathrm{XII}{ }^{\mathrm{e}}$ siècle, contenait toutes sortes de travaux mathématiques, aussi bien que les dernières parutions de l'époque, tel le Dialogus contra Iudaeos de Pierre Alphonse (vers 1109), œuvre importante pour la compréhension médiévale de l'Hébraïsme et de l'Islam. Hereford semble avoir été l'un des points de raccrochement de l'Angleterre à la translatio studii que d'autres clercs développaient dans la péninsule Ibérique et dans le royaume normand sicilien. Nous ne connaissons pas bien, cependant, ce rassemblement de savants anglais du XII ${ }^{\mathrm{e}}$ siècle. Lorsque l'on parle de Hereford, on met toujours en exergue la célèbre mappa mundi conçue vers 1300, aboutissement d'un penchant pour la géographie qui s'est manifesté très tôt dans cette école-cathédrale, et la figure de l'évêque Guillaume de Vere, grand savant et mécène de son temps pour les textes latins et vernaculaires ${ }^{10}$. Bien avant de devenir évêque, Guillaume de Vere a été patron d'un certain Gilbert, bouteiller, lequel a été à son tour commanditaire de Rouau d'Arundel pour sa traduction de la Lettre du Prêtre Jean, un poème anglo-normand qui témoigne manifestement d'une passion pour les merveilles des pays lointains. C'est au temps de l'épiscopat de Guillaume de Vere à Hereford que l'on peut situer l'existence de l'école autour de la cathédrale. De cette école faisaient probablement partie Roger Infans, Daniel de Morley, Alfred de Sareshel (ou Alfred l'Anglais), Robert Grosseteste et tant d'autres savants ${ }^{11}$.

L'histoire de l'établissement est pour autant énigmatique. Il paraît que certaines œuvres du maître Roger Infans avaient été commencées bien

9. Hue De Rotelande, Ipomedon, p. 8-11.

10. Pour plus de détails, voir J. BARROw, «A Twelfth-Century Bishop and Literary Patron : William de Vere », Viator, 18 (1987), p. 175-189.

11. Voir sur ce sujet l'ancienne étude de J. C. RusSELL, « Hereford and Arabic Science in England about 1175-1200 », Isis, 18/1 (1932), p. 14-25, qui contient certainement quelques exagérations, mais dont les conclusions demeurent globalement pertinentes. 
avant l'arrivée de Guillaume de Vere à Hereford. Maître Roger avait écrit son Comput (1176) pour Gilbert Foliot alors que ce dernier était évêque de Londres (1163-1187), mais Gilbert Foliot avait également été l'un des évêques de Hereford (1148-1163). Alfred de Sareshel, un autre savant de Hereford, a visité la péninsule Ibérique, d'où il a certainement ramené plusieurs textes savants, en particulier des traductions d'Aristote dont il s'est servi pour ses écrits ${ }^{12}$. En dernier lieu, ce serait toujours à Hereford que Robert Grosseteste aurait pris contact pour la première fois avec les œuvres arabes ${ }^{13}$. Ce qui se produisait dans cette école à Hereford avait peu d'échos par rapport aux grands mouvements culturels de la Renaissance $\mathrm{du} \mathrm{XII}^{\mathrm{e}}$ siècle. Néanmoins, c'était quelque chose d'important pour la vie culturelle de l'Angleterre. Les savants de cette ville voisine du Pays de Galles avaient des contacts avec le milieu culturel de l'Espagne et de l'Italie du Sud. Peut-être connaissaient-ils, à Hereford, les œuvres (ou le nom) du rhéteur grec du temps de l'Antiquité ?

Les œuvres d'Hermogène de Tarse étaient lues et copiées en Calabre, au XII ${ }^{\mathrm{e}}$ siècle, c'est-à-dire dans le pays même où se passe l'action du roman de Hue de Rotelande. Le ms. grec 3032 de la BnF contient plusieurs œuvres anonymes, accompagnées par les Progymnasmata d'Aphtonios d'Antioche, par des fragments d'Athanase d'Alexandrie et de Basile de Césarée, par quatre œuvres d'Hermogène de Tarse (Progymnasmata artis rhetoricae, Ars rhetorica de partitione statuum, Methodus de apto et solerti dicendi genere et les Scholia in Hermogenem), par les lettres d'Hippocrate et d'Artaxerxès, ainsi que par d'autres textes de rhétorique, attribués à Maxime de Tyre, Phoebammon ou Théodose. Le codex a été transcrit au $\mathrm{X}^{\mathrm{e}}$ siècle en Calabre ${ }^{14}$. Santo Lucà le compare à un autre manuscrit, de Messine, datant du XII ${ }^{\mathrm{e}}$ siècle (Paris, BnF, grec 119), lequel contient cette fois-ci un commentaire d'une œuvre d'Hermogène ${ }^{15}$. Enfin, pour Daniele Arnesano, l'étude de la rhétorique devait être rare, sinon exceptionnelle, en Italie du Sud ${ }^{16}$. Le ms. grec 3032 de la BnF constituerait donc un exemple

12. Pour toutes ces informations, voir D. MeTLITZKI, The Matter of Araby in Medieval England, New Haven/Londres, 1977, p. 38-41, qui a repris l'argumentation de J. C. Russell et a parlé de manière encore plus détaillée de cette école savante peu connue.

13. R. W. SOUTHERn, Robert Grosseteste : The Growth of an English Mind in Medieval Europe, Oxford, 1986, p. 66-68 ; J. McEvoY, Robert Grosseteste, Oxford, 2000, p. 28.

14. Pour ce ms., voir en dernier lieu C. FÖRSTEL et M. RASHED, «Une rencontre

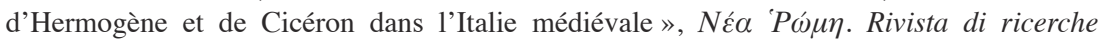
bizantinistiche, 3 (2006), p. 361-371.

15. S. LuCÀ, «Note per la storia della cultura greca della Calabria medioevale », Archivio storico per la Calabria e la Lucania, 74 (2007), p. 43-101 (p. 57).

16. D. ARNESANO, «Ermogene e la cerchia erudita. Manoscritti di contenuto retorico in Terra d'Otranto », dans N. BIANCI éd., La tradizione dei testi greci in Italia meridionale. Filagato da Cerami philosophos e didaskalos. Copisti, lettori, eruditi in Puglia tra XII e XVI secolo, Bari, 2011, p. 95-111 (p. 101-102, note 61). 
isolé, mais il peut toutefois prouver que la lecture des rhétoriciens antiques, quoique peu courante, était un phénomène culturel qui avait pu se produire dans le milieu savant de la Calabre. Si Hereford était en contact avec le milieu savant du royaume normand méridional, le nom d'Hermogenés devrait certainement venir de là.

À mon avis, Hue de Rotelande, auteur du XII ${ }^{\mathrm{e}}$ siècle, était conscient des préoccupations savantes de ses contemporains, d'autant plus que l'un d'eux, Simon de Freine, un autre clerc de Hereford, était lui-même un auteur de langue française et latine ${ }^{17}$. Ce que nous contemplons à Hereford, c'est sans doute un milieu composite, avec les grands savants de l'écolecathédrale, mais aussi avec des personnages tel Simon, auteur d'épîtres en vers adressées à Giraud de Barri aussi bien qu'auteur de deux traductions en vers français (la Vie de saint Georges et le Roman de Philosophie - une traduction de la Consolatio de Boèce). La place que pouvait occuper Hue de Rotelande dans ce milieu culturel devait être périphérique. C'était un auteur de romanz, ce n'était pas un grand savant. Il faisait pourtant partie de ce groupe et peu importe ici son véritable statut. De plus, la présence du nom d'Hermogenés, sans doute en rapport avec Hereford et l'Italie du Sud, n'est pas le seul témoin du fait que notre auteur avait pu emprunter le nom du rhéteur grec à ces sources. Une autre circonstance insolite peut être citée à ce propos.

Les deux romans de Hue de Rotelande ont pour cadre géographique les Pouilles et la Calabre, deux régions à la fois antiques et chevaleresques, comme dans les romans antiques du XII ${ }^{\mathrm{e}}$ siècle. C'est dans cette direction que l'on doit chercher pour expliquer la présence du nom d'Hermogenés dans le texte de l'Ipomedon. Néanmoins, la plupart des recherches consacrées à ce roman se sont concentrées, d'une part, sur les aspects misogynes, d'autre part, sur le côté parodique de l'œuvre littéraire, y compris sur les motifs et les noms que l'Ipomedon a empruntés à d'autres textes de son époque ${ }^{18}$. À

17. V. AgrigoroaeI, «Qu'y a-t-il dans un auteur ? Simund de Freine en dialogue avec Giraud de Barri », dans G. Borriero, R. CAPElli, C. Concina, M. SAlgaro et T. ZANON éd., Amb. Dialoghi e scritti per Anna Maria Babbi, Vérone, 2016, p. 145-153.

18. Voir à ce propos M. STANESCO, «Le secret de l'estrange chevalier: notes sur la motivation contradictoire dans le roman médiéval », dans G. S. BURGESS et R. A. TAYLOR éd., The Spirit of the Court : Selected Proceedings of the Fourth Congress of the International Courtly Literature Society (Toronto 1983), Cambridge, 1985, p. 339-349; W. CALIN, « The Exaltation and Undermining of Romance: Ipomedon », dans N. J. LACY, D. KELLY et K. Busby éd., The Legacy of Chrétien de Troyes, Amsterdam, 1988, vol. 2, p. 111-124 ; B. Hosington, « The Englishing of the Comic Technique in Hue de Rotelande's Ipomedon », dans J. BEER éd., Medieval Translators and Their Craft, Kalamazoo, 1989, p. 247-263 ; W. CALIN, « Contre la fin'amor ? Contre la femme ? Une relecture de textes du Moyen Âge », dans K. Busby et E. KoOPER éd., Courtly Literature : Culture and Context. Selected Papers from the 5th Triennial Congress of the International Courtly Literature Society, Dalfsen, The Netherlands, 9-16 August, 1986, Amsterdam/Philadelphie, 1990, p. 61-82 ; S. MuLA, " "Le bel teisir est curteisie". Il segreto come chiave di lettura dell'Ipomedon di Hue de Rotelande », 
ce propos, on aurait pu s'attendre à ce qu'il emprunte aussi quelque chose à l'histoire événementielle, surtout quand la terre de Calabre livresque était imaginée en rapport avec une autre Calabre, réelle et historique, l'un des pays de la diaspora normande des $\mathrm{XI}^{\mathrm{e}}-\mathrm{XII}{ }^{\mathrm{e}}$ siècles. Or, les études sociohistoriques n'ont pas observé que le nom d'un héros de cette Italie normande était caché dans un jeu de mots de l'Ipomedon. Plus précisément, il s'agit de la métamorphose du célèbre croisé Bohémond en chien de chasse.

\section{Bohémond le bâilleur}

Dans le roman, Ipomedon se déguise souvent et tient des discours à double entente. Il se déguise pour participer à un tournoi de trois jours et prétend qu'il part en réalité à la chasse. Le premier soir, Ipomedon se fait raconter ses propres exploits au tournoi par le chambellan Thoas. Celuici ignore ce que son interlocuteur a fait dans la journée. Ipomedon lui raconte à son tour que ses chiens Noblet, Ridel et Baucan ont bien couru, que Baucan a remporté le premier prix et qu'il a attrapé trois cerfs. Les cerfs représentent ici les trois chevaux des chevaliers vaincus. Plus tard, le deuxième soir, Ipomedon raconte les exploits cynégétiques du chien Ridel, autre substitut de lui-même. Et enfin, le troisième soir, notre protagoniste raconte les exploits du brachet noir Baailemunt, un chien qu'il n'avait pas nommé jusque-là. De nouveau, le chien est un substitut d'Ipomedon, car ce dernier avait porté une armure noire pendant le tournoi ${ }^{19}$ :

Ipomedon parole dunc.

«Esteez, amis, ne vus seit lunc,

Mes un petit parlez a mei ;

Dites de la meie part al rei,

Se estur eurent deske la nut,

Jo rai eü mut bel dedut,

Se tuz les ad vencuz li neir,

E jo vus redirrai tut veir,

dans U. FloRIS et M. VIRDIS éd., Il segreto. Atti del Convegno di Studi (Cagliari, 1-4 aprile, 1998), Rome, 2000, p. 221-241 ; F. MoRA, « Remploi et sens...». Cf. R. L. KRUEGER, « Misogyny, Manipulation, and the Female Reader in Hue de Rotelande's Ipomedon », dans K. Busby et E. KoOPER éd., Courtly Literature ..., p. 395-409 ; R. L. KRUEGER, « Playing to the Ladies : Chivalry and Misogyny in Ipomedon, Le chevalier à l'épée, and La Vengeance Raguidel », dans EAD., Women Readers and the Ideology of Gender in Old French Verse Romance, Cambridge/New York/Melbourne, 1993, p. 68-100, qui s'intéresse surtout à l'antiféminisme de l'Ipomedon, afin d'observer qu'il est utilisé pour mettre en évidence les tensions de genre dans le discours chevaleresque.

19. Hue DE Rotelande, Ipomedon, v. 6505-6522, p. 352-353. Pour une synthèse et une interprétation de ce long passage, voir J. VINOT, Répétition et variation..., p. 173-174. 
Un men brachet neir Baailemunt

Mes chens venqui quanqe il sunt.

Amis, entendez ma resun,

Portez lui de la veneisun

E si dites mut ben al rei,

Ke en pece n'avra plus par mei,

Kar il meisme i pot ore entendre

E asez cerfs e bisses prendre ! »

Il dist veir, mes nel sout fors lui,

Ke en pece n'avra plus par lui.

Baailemunt n'apparaît qu'une seule fois dans le roman, pour un banal effet comique. Il ne fait pas partie de la liste des chiens d'Ipomedon que celui-ci nomme dans les discours qu'il avait tenus les soirs précédents, ce qui permet d'entendre que l'utilisation du nom avait une valeur ponctuelle ${ }^{20}$. «Baailemunt » n'est alors qu'un ornement dans la narration ${ }^{21}$. Il s'agit d'une allusion par équivoque qui devait rappeler aux Normands d'Angleterre les exploits de leur compatriote méridional, le croisé devenu prince d'Antioche ${ }^{22}$.

Marie-Luce Chênerie a analysé les noms propres dans les deux romans de Hue de Rotelande. Elle explique l'utilisation parodique de certains noms :

20. Les trois chiens d'Ipomedon sont présentés dans son discours de la première soirée : «Mut ad ui ben curu Nublet, / E Ridel e tuit mi brachet, / Si ke treis granz cerfs ai hui pris ; / Mes de trestuz mes chens le pris, / De ceus ke geui unt curu, / Enporte Baucan le velu, / Li autre se sunt mut penez, / Mes il les ad trestuz passez » (HuE DE Rotelande, Ipomedon, v. 4425-4432, p. 263). Des trois chiens mentionnés dans ce premier passage, le nom de Baucan réapparaît aux v. 4465-4466, immédiatement après la première scène (« Cum ses chens unt curu le jur / E ke Baucan fu le meillur »), et celui de Ridel aux v. 5473-5474 (« Hui unt mes chens mut ben curru, / Mes Ridel les ad tuz vencu ») (HuE DE Rotelande, Ipomedon, v. 4465-4466, p. 265 et v. 5473-5474, p. 308).

21. De manière analogue, le nom du chien "Baucan" parodie celui d'un destrier célèbre (cf. J. VINOT, Répétition et variation..., p. 173). Il s'agit du destrier de Guillaume dans Aliscans ("Baucent").

22. L'histoire de Bohémond, de son père Robert Guiscard et du reste des Hauteville suditaliques a également fait l'objet des textes en langue vernaculaire. Voir à ce propos L'estoire de Tangré d'Oteviller dans les mss. Paris, BnF, fr. 17177, f. 267v-268r (incomplet de la fin, daté du XIII ${ }^{\mathrm{e}}$ siècle) ; Berne, Burgerbibliothek, Litt. 113, f. 166v-169v (avant 1270) ; Paris, BnF, n. a. fr. 3537 (copie du ms. de Berne, XVIII ${ }^{e}$ siècle) ; et Paris, BnF, fr. 24431, f. 53r et $54 \mathrm{r}$ (acéphale et incomplet de la fin, daté du XIII ${ }^{\mathrm{e}}$ siècle). Ce texte a été édité de manière maladroite par Francisque Michel dans Les Chroniques de Normandie, éd. F. Michel, Rouen, 1839, p. 1-4. Cf. P. MEYER, « Notice du manuscrit fr. 17177 de la Bibliothèque nationale (Histoire universelle ; Brut en prose ; poésies et chroniques diverses) », Bulletin de la Société des anciens textes français, 21, 1895, p. 80-118 (p. 110-112). 
Même la dénomination des personnages secondaires n'a pas laissé Hue indifférent ; celle-ci peut en effet contribuer momentanément à l'ironie ou au burlesque du projet d'ensemble; et puis la métaphore première est abandonnée avec une joyeuse désinvolture, quand le personnage, proche du protagoniste par sa fonction, est en fait voué à la polyvalence d'un auxiliaire $^{23}$.

C'est exactement le cas du chien Baailemunt, qui n'apparaît qu'une seule fois dans le récit, afin de condenser, me semble-t-il, un rire ponctuel : Bohémond - un chien bâillant.

Il se peut donc que Hue de Rotelande ait décidé de parodier non seulement le roman chevaleresque ou les romans antiques, mais aussi le penchant que les savants de Hereford avaient pour 1'Italie du Sud ${ }^{24}$. Les équivoques se multiplient et s'empilent dans son histoire: Bohémond est un chien, le chien est Ipomedon; Ipomedon devient Bohémond. Pareillement, puisque tout plonge dans le dérisoire, le père du héros bâilleur est Hermogène de Tarse, grand rhétoricien. On a l'impression que l'auteur se moque de tout : de l'histoire, de la science, de la littérature, de la vulgarisation. Mais nous sommes au tournant du XIII ${ }^{\mathrm{e}}$ siècle. Avec Hue de Rotelande, nous sommes loin des salons de la bourgeoisie du XVIII ${ }^{\mathrm{e}}$ siècle et de l'Öffentlichkeit de Jürgen Habermas ${ }^{25}$. Il n'y avait pas de véritable vulgarisation du savoir scientifique non plus, ni de « familiarisation de la science ». Il y avait en revanche quelque chose de différent.

\section{Hue de Rotelande et l'École de Hereford}

Par rapport aux grandes villes européennes de la fin $\mathrm{du} \mathrm{XII}{ }^{\mathrm{e}}$ siècle, Hereford comptait peu d'habitants. Les murs de cette ville étaient en train d'être édifiés, de même que le palais de l'évêque. C'était un milieu dynamique et il est fort probable que les nobles locaux, venus rencontrer l'évêque, connaissaient les savants, d'autant plus que ces derniers étaient souvent membres du chapitre. Il est aussi possible de supposer que les nobles entretenaient des conversations avec les chanoines et qu'ils apprenaient,

23. M.-L. CHÊNERIE, « Dénomination et anthroponymie... », p. 229.

24. Il y a là deux niveaux parodiques profondément différents : d'une part, la parodie de textes connus, dont le lecteur médiéval pouvait se rendre compte et qu'il pouvait goûter ; d'autre part, un clin d'œil à des clercs, qui peut-être ne le lisaient pas. Le fait qu'il existe un lien ne signifie pas que le lecteur médiéval soit en mesure de le reconnaître ou de l'identifier, ni même que d'autres clercs y parviennent. Je dirais que si preuve il y a, elle se situe du côté de l'intention de l'auteur, et non pas de sa réception.

25. J. Habermas, L'Espace public. Archéologie de la publicité comme dimension constitutive de la société bourgeoise, trad. M. B. DE LAUNAY, Paris, 1978. 
ponctuellement, quelles étaient les préoccupations des maîtres. Or, il apparaît que notre auteur connaissait ces deux catégories de personnes : d'une part, il écrivait pour les nobles ; d'autre part, il était familier avec au moins deux des savants de Hereford (Gautier Map et Hugues de Hongrie). Il est tout à fait probable que les nobles connaissaient aussi les savants. Le monde était petit.

À mon avis, le surgissement d'Hermogenés et de Bohémond dans le texte de l'Ipomedon témoigne de plaisanteries privées, dont la vraie signification n'est connue que des amis qui en sont les destinataires. Parmi ces destinataires, il y en a eu qui n'ont pas nécessairement goûté l'humour de Hue de Rotelande. Certains étaient inévitablement trop sérieux pour s'amuser avec des histoires de chiens, de chasse et de tournois. Roger Infans ou Alfred de Sareshel, grands savants, n'avaient pas le temps pour ce genre de divertissements. D'autres, en revanche, avaient développé un goût pour les histoires moins sérieuses. Gautier Map, non moins savant que les deux premiers, s'intéressait aux balivernes des courtisans. Il partageait avec Hue un « esprit mordant et irrévérencieux ${ }^{26}$ ». Quant à Simon de Freine, il était quelqu'un de grave, mais il s'amusait à échanger hexamètres et distiques élégiaques avec un autre ami, éloigné, Giraud de Barri. Qui plus est, Simon écrivait des romanz hagiographiques ou philosophiques en français. C'était un savant, mais un savant qui faisait aussi une sorte de vulgarisation. Il y avait de tout dans cette École de Hereford: des sages sévères, des poètes sérieux, de beaux esprits, des vulgarisateurs, mais aussi des moqueurs.

Hue de Rotelande n'était sans doute pas l'un des membres permanents du groupe. Il vivait à neuf kilomètres de la ville, il n'était pas si instruit que les autres, mais il appartenait en quelque sorte à ce milieu. Hue avait inclus le nom d'un des sages de Hereford dans son poème : il s'amusait en suggérant que le chanoine Hugues de Hongrie, une personne âgée, aurait connu les arts de la séduction ${ }^{27}$. Il disait également qu'il n'était pas le seul à raconter des mensonges ; Gautier Map l'avait déjà fait ${ }^{28}$. Hue de Rotelande jouait donc le rôle d'un Hugo Dyson ridiculisant les écrits de J. R. R. Tolkien dans

26. HUE DE RotelANDE, Ipomedon, p. 9 pour la citation.

27. «Quant la reïne aveit mangé, / E li chevaler sunt descé, / Sis druz en la chambre de la meine, / Si la besa de bon' estraine; / Cument ke il fust, a la reïne, / Fust le beser bone mecine, / Mes il le prist trestut a gabs. / Certes, jo nel fereie pas, / Einz i mettreie mut grant peine, / Tant ke tastee fust la veine / Par unt le mal si la teneit ; / Huge de Hungrie par dreit / s'en deüst mut ben entremetre, / La glose set de ceste lettre » (HUE DE RoTELANDE, Ipomedon, v. 5509-5522, p. 310). L'obit de Hugues de Hongrie figure deux fois dans les registres de la cathédrale de Hereford ; le chanoine Hugo de Hungaria apparait également en tant que témoin d'une charte de Hereford en 1153-1155 (cf. HUE DE ROTELANDE, Ipomedon, p. 11, n. 11). L'âge du personnage (plus que soixante-dix ans) explique l'astuce de Hue de Rotelande.

28. « Sul ne sai pas de mentir l'art, / Walter Map reset ben sa part » (HuE DE RotelandE, Ipomedon, p. 380, v. 7185-7186). 
les réunions des Inklings oxoniens. Quand on le regarde à la lumière de la littérature de son temps, le burlesque de l'Ipomedon n'est pas très différent de la réplique de Dyson : « ... no more elves! »

\section{Vladimir Agrigoroaei - CÉSCM Poitiers-CNRS}

\section{Hermogène le rhéteur et Bohémond le bâilleur. Échos de l'École de Hereford dans I'Ipomedon de Hue de Rotelande}

L'Ipomedon de Hue de Rotelande reprend des thèmes et des motifs d'autres textes littéraires, mais il demeure esthétiquement singulier. Le présent article observe que les noms de deux personnages de ce roman parodique en vers de la fin du XII siècle (le roi " Hermogenés " et le chien " Baailemunt ") constituent des allusions aux textes ou aux histoires ayant des auteurs ou des protagonistes en Italie du Sud. Ces échos sud-italiques seraient dus au fait que l'auteur du roman vivait dans le voisinage de Hereford, à une époque où les clercs de cette ville entretenaient des contacts avec le milieu savant de l'Italie méridionale.

Anglo-normand, Hereford, Hermogène de Tarse, Hue de Rotelande, Ipomedon, Italie du Sud, onomastique.

\section{Hermogenes the Rhetorician and Bohemond the Yawner. Echoes from the Hereford School in Hue de Rotelande's Ipomedon}

The romance of Ipomedon by Hue de Rotelande borrows certain themes and motifs from other literary texts, all the while remaining aesthetically unusual. This article notes that the names of two characters in this late twelfth-century verse parody (the king "Hermogenes" and the dog "Baailemunt") constitute allusions to texts or stories with authors or protagonists in southern Italy. These southern Italian echoes are due to the fact that the author of the novel lived close to Hereford, at a time when the clerics of this city maintained contacts with the academic circle of southern Italy.

Anglo-Norman, Hereford, Hermogenes of Tarsus, Hue de Rotelande, Ipomedon, Onomastics, Southern Italy. 
